'Departamento de Cirugía Vascular y Endovascular, Pontificia Universidad Católica de Chile. Santiago, Chile.

Trabajo Financiado con Fondos del Departamento de Cirugía Vascular y Endovascular, Pontificia Universidad Católica de Chile. Los autores declaran no tener conflictos de interés

Recibido el 4 de junio de 2020, aceptado el 30 de noviembre de 2020.

Correspondencia a: Dr. Leopoldo Mariné $\mathrm{M}$. Apoquindo 3990 Oficina 601. Las Condes, Santiago, Chile. Zip Code: 7550112 marinepolo@yahoo.com

\section{Aneurisma de aorta abdominal roto al duodeno: causa infrecuente de hemorragia digestiva masiva}

\author{
LEOPOLDO MARINE ${ }^{1}$, RENATO MERTENS ${ }^{1}$, \\ IGNACIO TORREALBA ${ }^{1}$, FRANCISCO VALDÉS ${ }^{1}$, \\ MICHEL BERGOEING ${ }^{1}$, FRANCISCO VARGAS ${ }^{1}$, HUGO YÁÑEZ ${ }^{1}$
}

\section{Rupture of abdominal aortic aneurysm into the duodenum: uncommon cause of massive gastrointestinal bleeding}

Primary aortoenteric fistula is the spontaneous communication between the lumen of the aorta and a portion of the digestive tract. The most common cause is the erosion of an abdominal aortic aneurysm into the $3^{\text {rd }}$ or $4^{\text {th }}$ portion of the duodenum. It manifests clinically as gastrointestinal bleeding, with or without abdominal pain and a pulsatile abdominal mass on physical exam. Gastrointestinal bleeding is initially recurrent and self-limiting and progresses to fatal exsanguinating hemorrhage. Endoscopic examination diagnoses only $25 \%$ of aortoenteric fistulas because these are usually located in the distal duodenum. Contrast computed tomography of the abdomen and pelvis is diagnostic in only $60 \%$ of cases. We report three cases with this condition. A 67-year-old male presenting with an upper gastrointestinal bleeding. He was operated and a communication between an aortic aneurysm and the duodenum was found and surgically repaired. The patient is well. A 67-year-old male with an abdominal aortic aneurysm presenting with abdominal pain. He was operated and anticoagulated. In the postoperative period he had a massive gastrointestinal bleeding and a new CAT scan revealed an aorto enteric fistula that was surgically repaired. The patient is well. An 82-year-old male with an abdominal aortic aneurysm presenting with hematochezia. A CAT scan revealed a communication between the aneurysm and the third portion of the duodenum, that was surgically repaired. The patient died in the eighth postoperative day.

(Rev Med Chile 2021; 149: 132-136)

Key words: Gastrointestinal Hemorrhage: Aortic Aneurysm; Digestive System Fistula.
U na fístula aortoentérica (FAE) es la comunicación espontánea entre el lumen de la aorta y una porción del tubo digestivo ${ }^{1,2}$. Pese a haber sido descrita por Cooper hace casi 200 años, continúa siendo difícil de diagnosticar y de tratar, con alta morbimortalidad asociada ${ }^{3,4,5}$.

$\mathrm{Su}$ frecuencia es extremadamente baja, siendo $0,04-0,07 \%$ de las autopsias ${ }^{1,4,6}$. Se han reportado menos de 400 casos, siendo la mayoría reportes de casos únicos ${ }^{1,3,4,6-8}$ y escasas revisiones de casos acumulados ${ }^{2,5,9}$. Las FAE se clasifican en primaria, cuando existe una comunicación espontánea entre la aorta nativa, generalmente un aneurisma aórtico abdominal (AAA) y un segmento del tubo digestivo, y FAE secundaria, cuando existe cirugía aórtica previa, siendo 10 veces más frecuente que la FAE primaria ${ }^{4,6}$.

El diagnóstico de FAE primaria es un desafío 
clínico mayor por la ausencia del antecedente de cirugía aórtica previa. Sin un diagnóstico y reparación quirúrgica oportunos, la mortalidad es $100 \% \%^{1,3,4,6,8}$. El diagnóstico descansa en un alto índice de sospecha ante hemorragia digestiva $y$ presencia de un $\mathrm{AAA}^{3,7}$. La hemorragia digestiva es su síntoma cardinal, aunque corresponde solo a $0,2 \%$ de todas las causas de hemorragia digestiva ${ }^{1}$.

Presentamos nuestra experiencia en tres casos de FAE primaria y AAA infrarrenal tratados con cirugía abierta en los últimos 25 años.

\section{Caso clínico 1}

Hombre de 67 años ex fumador, tratado por hipertiroidismo, que consultó por cuadro de 12 días de evolución de dolor abdominal y lumbar asociado a melena. Al ingreso presentaba una masa abdominal pulsátil y sensible. Una ecografía abdominal evidenció un AAA de $40 \mathrm{~mm}$. Posterior al estudio presentó hipotensión grave, distensión abdominal y salida de abundante contenido hemático por sonda nasogástrica. Sin otros estudios, se intervino de urgencia, realizándose laparotomía media y gastrotomía, la que dio salida a sangrado abundante desde el duodeno. Presentó paro cardiorrespiratorio, recuperado con maniobras de resucitación. Se identificó una amplia comunicación entre el duodeno y el AAA, requiriendo control aórtico endoluminal con balón de sonda Foley y puente aorto-aórtico in situ con prótesis de dacrón, gastroduodenorrafia, yeyunostomía y parche omental. En el postoperatorio fue reoperado por peritonitis estercorácea secundaria a necrosis del colon, realizándose una hemicolectomía izquierda y colostomía terminal. Mantuvo tratamiento antibiótico durante su hospitalización de 38 días de duración. Se encuentra vivo y sin complicaciones a los 24 años de la cirugía.

\section{Caso clínico 2}

Hombre de 67 años, diabético tipo 2, fumador y con enfermedad diverticular. Consultó en otra institución por cuadro de $48 \mathrm{~h}$ de evolución de dolor abdominal difuso, intenso y persistente. Una tomografía axial computada (TAC) contrastada de abdomen y pelvis mostró un AAA sacular de 54 $\mathrm{mm}$ no complicado. Por persistencia de intenso dolor se realizó una laparotomía exploradora, reportándose aspecto isquémico del ciego y colon ascendente. Cerrada la laparotomía, fue anticoagulado por dicho hallazgo. En el decimosexto día postoperatorio el paciente presentó hipotensión súbita, dolor abdominal intenso y hematemesis de $300 \mathrm{ml}$. Se realizó endoscopía digestiva alta que reveló sangrado activo por lesión en la $3^{a}$ porción del duodeno, siendo trasladado a nuestro hospital. $\mathrm{Al}$ ingreso presentaba hematocrito de $26 \%$. Una TAC con contraste en fase arterial (AngioTAC) diagnosticó una fístula aorto-duodenal primaria, operándose de urgencia por laparotomía media. En ausencia de contaminación local, se realizó un puente aorto-aórtico in situ con prótesis de dacrón, resección del segmento duodenal comprometido (Figura 1) y anastomosis duodeno-yeyunal

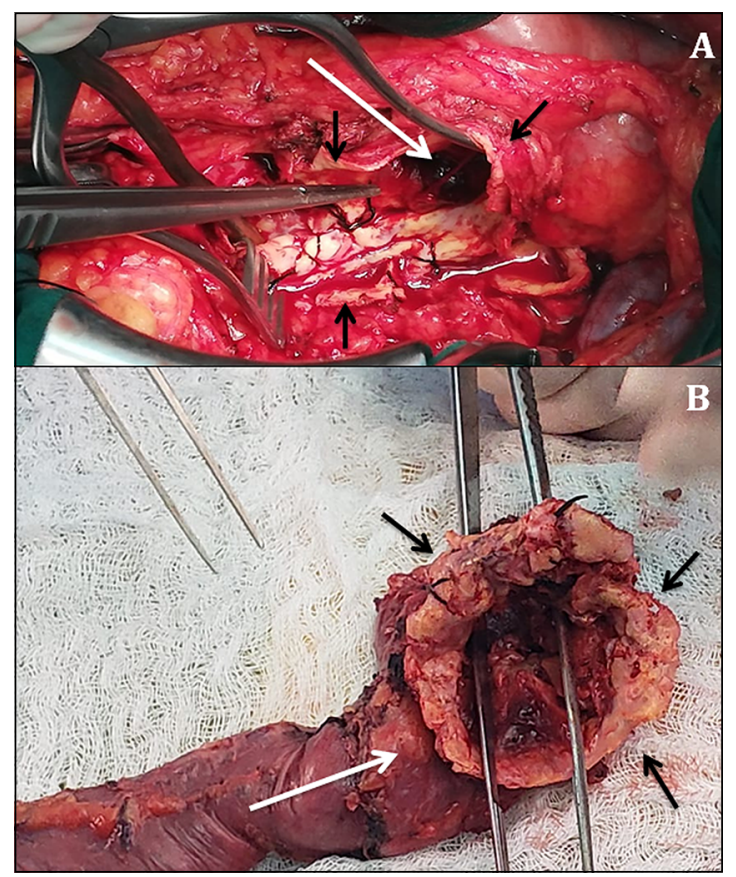

Figura 1. A: Imagen intraoperatoria de aorta abdominal aneurismática abierta longitudinalmente (flechas negras cortas). Se aprecia la presencia de coágulo que ocluye la fístula aorto-duodenal (flecha larga blanca). B: Imagen de pieza operatoria en la que se puede apreciar parte de la pared aórtica cortada transversalmente (flecha negras cortas) y tercera y cuarta porción del duodeno junto a segmento de duodeno adosado a la aorta (flecha blanca larga). Se observa, además, una pinza introducida en el trayecto fistuloso una vez retirado el coágulo, apreciándose su amplitud por el grado de apertura de las ramas de la pinza, correspondiendo a $20 \times 35 \mathrm{~mm}$. 
más parche omental. Evolucionó con infección de herida operatoria, tratada localmente y con antibióticos por 21 días, siendo dado de alta al decimocuarto día postoperatorio. Se encuentra vivo, asintomático y sin evidencia de infección a los 15 meses de la cirugía.

\section{Caso clínico 3}

Hombre de 82 años hipertenso, diabético tipo 2, fumador, con EPOC y antecedente de pancreatectomía córporocaudal por pancreatitis hacía más de 10 años, con gran hernia incisional. Ingresó a otro centro por cuadro de 2 días de evolución de dolor abdominal, sangrado digestivo bajo abun- dante e hipotensión. En AngioTAC de abdomen y pelvis se evidenció un AAA de $93 \mathrm{~mm}$ de diámetro con comunicación hacia la tercera porción del duodeno (Figura 2). Trasladado a nuestro hospital, ingresó hipotenso, con hematocrito de $23 \%$. Se abordó el AAA por vía retroperitoneal, reparándose con un puente aorto-aórtico in situ de dacrón. Posteriormente, por vía anterior se reparó el segmento intestinal comprometido con exclusión duodenal mediante gastroyeyunoanastomosis. Se inició tratamiento antibiótico. Evolucionó con una evisceración contenida que fue tratada de forma conservadora. Al séptimo día se trasladó a su centro de origen. Falleció al octavo día, no se practicó autopsia.
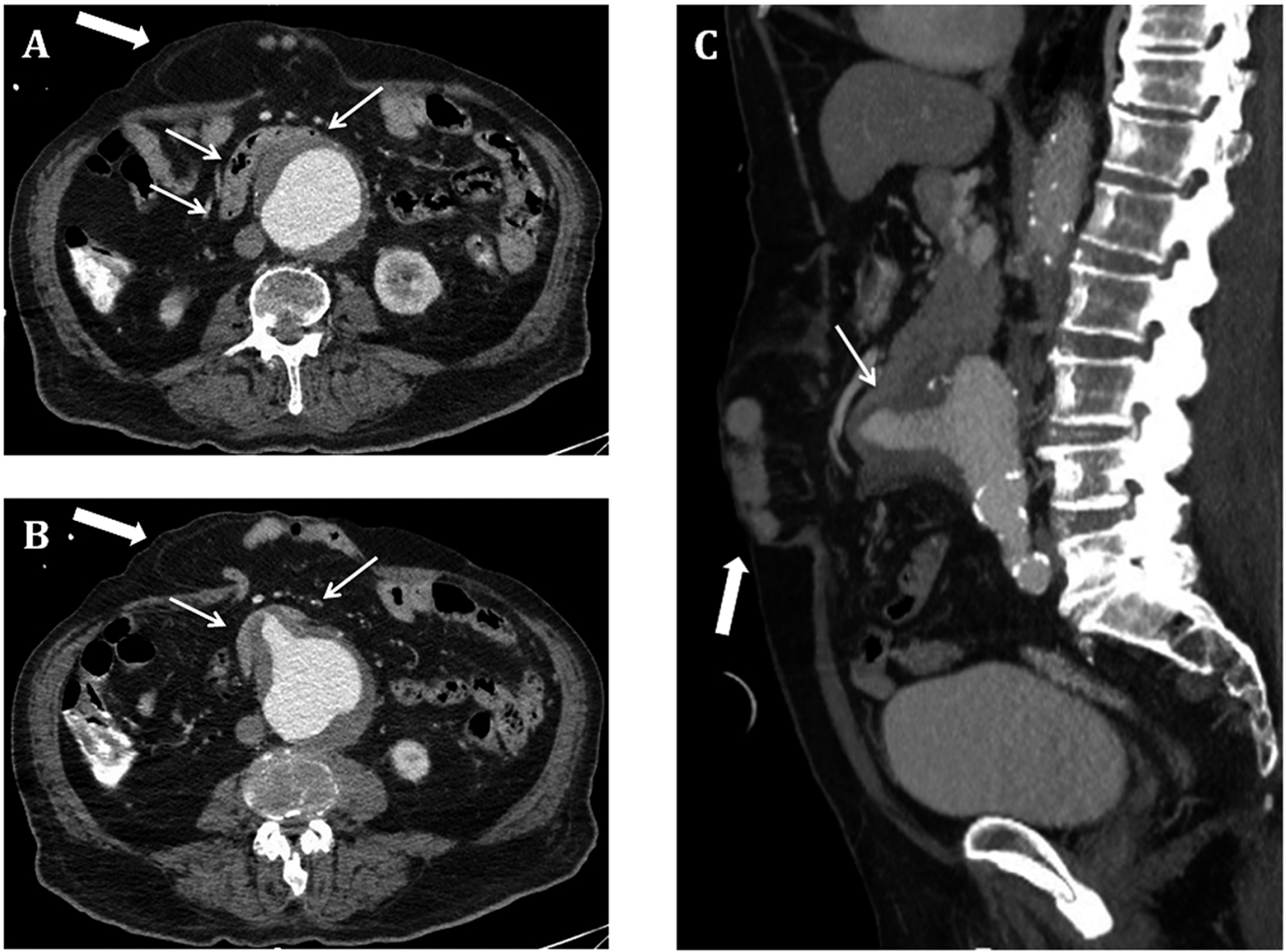

Figura 2. AngioTAC de abdomen y pelvis muestra una fístula aorto-duodenal primaria, en $\mathbf{A}$ y $\mathbf{B}$ se observan cortes transversales y en $\mathbf{C}$ un corte es sagital. Las imágenes muestran un aneurisma de aorta abdominal infrarrenal con trombo mural. La tercera porción del duodeno esta íntimamente en contacto con la aorta (flechas finas), estando ausente el plano de grasa entre la aorta y el duodeno. En $\mathbf{B}$ se observa una solución de continuidad del trombo mural apreciándose cercanía del contraste a la pared aórtica. Se observa, además, hernia incisional en línea media con contenido de epiplón y asas intestinales, lo que motivó acceso retroperitoneal. 


\section{Discusión}

De las FAE primarias, la fístula al duodeno corresponde a $75-93 \%$ de los $\operatorname{casos}^{1,2}$, comprometiendo con mayor frecuencia la tercera porción $(69 \%)$, seguido de la cuarta porción $(7 \%)^{4}$. En raras ocasiones, la FAE primaria puede ocurrir en aortas no dilatadas, por infección (aortitis séptica), ingesta de cuerpo extraño, radioterapia o tumores $^{4,6,9}$. Los tres pacientes reportados presentaban un AAA erosionado al duodeno.

La edad promedio de presentación en publicaciones es 63-64 años 5 ,9 , siendo más frecuente en hombres $(75-80 \%)^{2,7,9}$. Esta serie coincide con lo descrito. Desde el punto vista de la FAE como complicación de un AAA, esta ocurre en menos de $1 \%$ de los pacientes operados por AAA roto ${ }^{2}$, concordante con nuestra experiencia ${ }^{10}$.

La presentación clínica de la FAE primaria es inespecífica, debutando con hemorragia digestiva en el 64-94\% $\%^{4,6,8,9}$, dolor abdominal o lumbar $(32-48 \%)^{4,6,8,9}$ o masa abdominal pulsátil palpable $(17-25 \%)^{4,6,8,9}$. Esta tríada diagnóstica ha sido descrita en solo $10-25 \%{ }^{1,6}$ de los casos. Otras manifestaciones son shock hipovolémico $(33 \%)^{9}$, síncope $(10 \%)^{9}$, fiebre y sepsis ${ }^{4,5,6,8}$.

La hemorragia exanguinante suele ir precedida de uno o más episodios previos de sangrado leve y autolimitado, conocidos como hemorragia centinela. La hemorragia exanguinante se ha atribuido al desplazamiento del coágulo que ocluye la fístula ${ }^{1,3,6-8}$. En $33 \%$ de los casos la hemorragia masiva ocurrió dentro de las $6 \mathrm{~h}$ siguientes al episodio centinela ${ }^{1,8} y$ dentro de las $24 \mathrm{~h}$ en $50 \%{ }^{3}$. De nuestros pacientes, solo uno presentó la tríada diagnóstica, todos tuvieron hemorragia centinela y la hemorragia exanguinante ocurrió entre los 2 y 12 días después.

El diagnóstico precoz de FAE primaria es muy difícil debido a su baja prevalencia, ausencia de antecedentes relevantes, presentación insidiosa ${ }^{7}$ y clínica inespecífica ${ }^{6,8}$; razones que no permiten establecer un algoritmo diagnóstico. Sin embargo, puede plantearse en hombres mayores de 60 años con hemorragia digestiva y con antecedente de un AAA o hallazgo de masa abdominal pulsátil al examen físico ${ }^{8}$.

La dificultad diagnóstica obliga a realizar estudios complementarios. En pacientes estables, la endoscopía alta y la AngioTAC de abdomen y pelvis son recomendados. La endoscopía es el primer examen a realizar en toda hemorragia digestiva, sin embargo, en las FAE primarias el rendimiento diagnóstico es solo $25-30 \%^{1,3,4,9}$ : se observa el sitio del sangrado, un coágulo adherente al duodeno o la compresión extrínseca pulsátil por el $\mathrm{AAA}^{4}$. La endoscopía permite descartar causas habituales de hemorragia digestiva ${ }^{6,9}$ y tiene el riesgo teórico de desencadenar una hemorragia masiva al lavar el coágulo que contiene a la fístula ${ }^{7}$.

El rendimiento diagnóstico de la TAC con contraste en FAE primarias es $60 \%{ }^{7}$, con sensibilidad de $50-94 \%$ y especificidad de $85-100 \%{ }^{3,8}$. El signo patognomónico es el paso de contraste hacia el lumen intestinal. También son sugerentes: desaparición del plano adiposo entre aorta y duodeno, engrosamiento de la pared duodenal y aire ectópico en la pared aórtica o en el trombo mural ${ }^{1,3,6,8}$. Además, aporta información anatómica de la aorta ${ }^{1}$.

Pese a la utilización de estos métodos de estudio, en 50-66\% de los casos el diagnóstico ha sido en el intraoperatorio ${ }^{1,3,4}$, como aconteció en nuestro primer paciente. Ante inestabilidad hemodinámica, la exploración quirúrgica inmediata es mandatoria, ya que 5-16\% de los pacientes fallecen antes de ser trasladados a pabellón ${ }^{7,9}$.

La intervención es la única opción terapéuti$\mathrm{ca}^{6,9}$. La cirugía a realizar depende de la etiología, localización de la fístula y de la sospecha de contaminación retroperitoneal ${ }^{4}$. La técnica comprende la reparación del aneurisma, la reparación del duodeno e interposición de omento ${ }^{3}$. En ausencia de contaminación, es factible realizar una reconstrucción aórtica protésica in situ $u^{4}$, siendo la cirugía realizada con mayor frecuencia $\left(66 \%{ }^{9}\right)$, con una mortalidad de 30-34\% ${ }^{9}$. Esta fue la cirugía realizada en nuestros casos. Otras alternativas quirúrgicas son la ligadura aórtica y puente extraanatómico necesaria ante contaminación local evidente $^{4,6,9}$, con mayor mortalidad (40-90\%) . El tratamiento endovascular es una alternativa ante el alto riesgo quirúrgico o situaciones anatómicas adversas $^{1,3,6}$, pero tiene elevada tasa de infección protésica recurrente al no reparase el duodeno ${ }^{2}$. Puede ser considerado como un procedimiento de salvataje para, en un segundo tiempo, realizar un procedimiento definitivo ${ }^{11}$.

En el postoperatorio se requiere el uso de antibióticos empíricos por 7-10 días, debiendo prolongarse según resultado de cultivos o ante evidencia de infección ${ }^{9}$. El pronóstico global está 
definido por lo precoz del diagnóstico, la gravedad del sangrado y el compromiso hemodinámico asociado, el grado de contaminación o infección y la técnica de reparación utilizada ${ }^{2}$.

\section{Conclusión}

La FAE primaria es una condición muy infrecuente y grave, al igual que el antecedente de reparación aórtica en las FAE secundarias, la presencia de un AAA debiera alertar al clínico de esta posibilidad. La sobrevida del paciente depende del diagnóstico oportuno y el manejo quirúrgico precoz.

\section{Referencias}

1. Lin TC, Tsai CL, Chang YT, Hu SY. Primary aortoduodenal fistula associated with abdominal aortic aneurysm with presentation of gastrointestinal bleeding: a case report. BMC Cardiovasc Disord 2018; 18 (1): 113.

2. Rodrigues dos Santos C, Casaca R, Mendes de Almeida JC, Mendes-Pedro L. Enteric Repair in Aortoduodenal Fistulas: A Forgotten but Often Lethal Player. Ann Vasc Surg 2014; 28(3): 756-62.

3. Varghese M, Jorgensen GT, Aune C, Bergan R, Norderval S, Moland J. Primary Aortoduodenal Fistula - A Case Report and a Review of the Literature. Ann Vasc Surg
2016; 34: 271

4. Lemos DW, Raffetto JD, Moore TC, Menzoian JO. Primary aortoduodenal fistula: A case report and review of the literature. J Vasc Surg 2003; 37(3): 686-9.

5. Tareen AH, Schroeder TV. Primary Aortoenteric Fistula: Two New Case Reports and a Review of 44 Previously Reported Cases. Eur J Vasc Endovasc Surg 1996; 12(1): 5-10.

6. Shehzad KN, Riaz A, Meyrick-Thomas J. Primary aortoduodenal fistula - a rare clinical entity. JRSM Short Rep. 2010; 1(1): 7 .

7. Richards CR, McMurray R, Criman E, Rinehart S. Primary aortoduodenal fistula: a rare entity with lethal effects. BMJ Case Rep 2016 Oct 24; 2016. pii: bcr2016217001. doi: 10.1136/bcr-2016-217001.

8. Ishimine T, Tengan T, Yasumoto H, Nakasu A, Mototake H, Miura Y, Kawasaki K, Kato T. Primary aortoduodenal fistula: A case report and review of literature. Int J Surg Case Rep 2018; 50: 80-3.

9. Saers SJ, Scheltinga MR. Primary Aortoenteric Fistula. Br J Surg 2005; 92(2): 143-52.

10. Marine L, Valdes F, Mertens R, Kramer A, Vargas F, Bergoeing M, Torrealba JI, Urbina J. Open Surgery for Ruptured Abdominal Aortic Aneurysm - 38 Years Experience at an Academic Center in Chile. Ann Vasc Surg 2020; 64: 71-9.

11. Danneels MI, Verhagen HJ, Teijink JA, Cuypers P, Nevelsteen A, Vermassen FE. Endovascular Repair for Aorto-Enteric Fistula: A Bridge Too Far or a Bridge to Surgery? Eur J Vasc Endovasc Surg 2006; 32(1): 27-33. 